\title{
Prognostic Value of Stimulated Thyroglobulin Levels at the Time of Radioiodine Administration in Differentiated Thyroid Cancer
}

\author{
Mathieu Spaas $^{\mathrm{a}}$ Brigitte Decallonne $^{\mathrm{b}}$ Annouschka Laenen ${ }^{\mathrm{c}}$ Jaak Billen ${ }^{\mathrm{d}}$ \\ Sandra Nuyts ${ }^{a}$ \\ ${ }^{a}$ Department of Radiation Oncology, KU Leuven - University of Leuven, University Hospitals Leuven, Leuven, \\ Belgium; 'b Department of Endocrinology and Internal Medicine, KU Leuven - University of Leuven, University \\ Hospitals Leuven, Leuven, Belgium; ' Leuven Biostatistics and Statistical Bioinformatics Centre, Leuven, Belgium; \\ ${ }^{\mathrm{d}}$ Department of Laboratory Medicine, University Hospitals Leuven, Leuven, Belgium
}

\section{Keywords}

Thyroglobulin · Radioiodine administration · Differentiated thyroid cancer

\begin{abstract}
Objective: The prognostic value of stimulated thyroglobulin (sTg) and Tg-related parameters prior to and immediately after radioactive iodine (RAl) administration was assessed in a cohort of patients presenting with differentiated thyroid cancer (DTC) as a predictor of recurrent or progressive structural disease. Methods: Clinical records of 180 DTC patients were retrospectively reviewed, and serum TSH, $\mathrm{Tg}$, and $\mathrm{Tg}$ antibodies were recorded just before RAI administration (pre-) and at the time of whole body scanning (post-). Based on the results of initial staging and RAI scintigraphy, patients were divided into two groups: those who were considered to be structurally disease-free after thyroidectomy and RAI (group 1) and those who were not (group 2). Univariate analyses were performed for pre-Tg, ratioTg (post$\mathrm{Tg} / \mathrm{pre}-\mathrm{Tg}$ ), and other clinical and pathological markers for long-term outcome, as well as separate bivariate analyses focusing on pre-Tg to correct for possible confounders. Dif-
\end{abstract}

ferent pre-Tg cut-off values for predicting structural disease recurrence were assessed in a subgroup of patients in group 1 prepared with thyroid hormone withdrawal. Results: In group 1, $(n=166)$ male gender, higher T-stage and both Tgrelated parameters proved to be significant risk factors for structural disease relapse. Of all candidate variables, only higher T-stage served to predict progressive structural disease in group $2(n=14)$. Subgroup analysis showed a negative predictive value of $91.67 \%$ for pre-Tg $<10 \mu \mathrm{g} / \mathrm{L}$. Conclusion: The sTg value at the time of RAl administration may be helpful in predicting structural disease recurrence in patients with DTC. Published by S. Karger AG, Basel

\section{Introduction}

The vast majority (>90\%) of thyroid carcinomas are differentiated thyroid cancer (DTC), which includes papillary (PTC) and follicular cancer (FTC), characterized by an indolent course and low mortality [1]. Primary management of DTC usually consists of total thyroidectomy

\section{KARGER}

(c) 2018 European Thyroid Association

Published by S. Karger AG, Basel
Sandra Nuyts

Herestraat 49

BE-3000 Leuven (Belgium)

E-Mail sandra.nuyts@ uzleuven.be 
followed by radioactive iodine (RAI) remnant ablation (RRA). Postoperative administration of RAI serves three major goals: the first is to destroy any residual microscopic disease as well as any remaining normal thyroid tissue; secondly, RRA allows for serum thyroglobulin (Tg) to be used as a specific tumor marker and increases sensitivity of diagnostic whole-body iodine scintigraphy (WBS) in the follow-up, allowing early detection of recurrent or metastatic disease; a third and final advantage is that postablative WBS is superior to diagnostic RAI scanning in discovering regional or distant metastases [2].

Despite its excellent disease-specific survival rates, up to one-third of DTC patients may experience a clinically significant recurrence during long-term follow-up [3]. An accurate initial risk appraisal is therefore crucial in these patients because it not only defines the need for postoperative RAI administration, but also allows formulation of an appropriate surveillance strategy. Features known to correlate with poorer prognosis are higher American Joint Committee on Cancer (AJCC) stage, advanced age, male sex, extrathyroidal extension, and certain histological subtypes [4], but many authors have also researched serum Tg measured just before RAI administration as a marker for predicting persistent or recurrent disease, with reported cut-off values ranging from 1.0 to $10.0 \mu \mathrm{g} / \mathrm{L}$ [5-8]. Due to its high negative predictive value (NPV) in this setting, preablation Tg has been suggested to be a useful and inexpensive tool for risk evaluation early on during and after initial treatment.

The aim of this study was to assess the prognostic value of stimulated $\mathrm{Tg}$ (sTg) and Tg-related parameters at the time of RAI administration in a cohort of patients presenting with DTC at a single referral center in Belgium.

\section{Subjects and Methods}

\section{Patient Selection and Grouping}

Clinical records of all patients who received RAI administration after total thyroidectomy for DTC at the University Hospital in Leuven (Belgium) between 1st January 2011 and 31st December 2015 were retrospectively reviewed. Approval was obtained from The Supervisory Committee on Medical ethics of the "Master in Medicine (Leuven)" program and patient consent was not required.

Exclusion criteria were as follows: (1) prior history of RAI administration; (2) incomplete laboratory results; (3) elevated anti$\mathrm{Tg}$ antibodies; and (4) age at diagnosis $<18$ years old. Finally, a total of 180 patients were included in our study.

Patients were divided into two groups: group 1 consisted of a total of 166 patients who were considered to be structurally disease-free after primary treatment (thyroidectomy followed by RAI), defined as negative RAI scintigraphy and/or faint RAI up- take in the thyroid bed on WBS. Fourteen patients who did not meet this criterion were included in group 2. Relapse-free survival and progression-free survival for group 1 and 2 , respectively, were correlated with non-Tg- and Tg-related variables to determine their prognostic value.

\section{Risk Stratification}

Our hospital has developed a postoperative risk stratification for RAI decision-making in patients with DTC, largely based on the American Thyroid Association (ATA) guidelines (online suppl. Appendix A; for all online supplementary material, see www. karger.com/doi/10.1159/000489849) [7]. Depending on clinicopathological tumor features, patients are divided into four risk categories. (1) Patients with PTC stage $\mathrm{pT} 1 \mathrm{aN} 0 / \mathrm{x}$, or $\mathrm{pTla}(\mathrm{m}) \mathrm{N} 0 / \mathrm{x}$ with all tumor foci $<5 \mathrm{~mm}$, as well as FTC stage pT1 without vascular invasion or a minimally invasive histology and $\mathrm{pT} 2$ without vascular invasion are classified as very low-risk. (2) A low-risk group is defined as patients diagnosed with PTC stage $\mathrm{pTla}(\mathrm{m})$ $\mathrm{N} 0 / \mathrm{x}$ with at least one tumor focus $\geq 5 \mathrm{~mm}$, stage pT1aNla or pT1b-2N0-1a/x, FTC stage pT2, minimally invasive histology stage pT3-4 without vascular invasion, and any minimally invasive histology pT stage with vascular invasion. (3) Patients with PTC showing either of the following characteristics are categorized as intermediate risk: stage $\mathrm{pT} 1-2 \mathrm{~N} 1 \mathrm{~b}, \mathrm{pT} 1-2 \mathrm{~N} 0-1 / \mathrm{x}$ with aggressive histology (e.g., tall cell, columnar cell), pT3N0, or pT4aN0$1 / x$. This also applies to any $\mathrm{pT}$ stage PTC with nodal involvement $>5$ nodes, $\geq 3$ nodes with positive section margins, or at least 1 node $>3 \mathrm{~cm}$, as well as to FTC stage pT3-4a and pT1-2 with aggressive histology (e.g., Hürthle cell). (4) If DTC stage pT4b or cM1 is present, patients are included in the high-risk group.

\section{RAI Protocol}

All patients had undergone a total or completion thyroidectomy, either at our institution or at a regional hospital. Histologic reports were written by the respective pathologists, with or without review by our institution's Pathology Department.

Based on the aforementioned risk categories, patients were selected for one of two possible TSH stimulation modalities prior to receiving RAI. Firstly, thyroid hormone withdrawal (THW) consisted of LT4 withdrawal during the 6 weeks leading up to RAI administration. LT4 was substituted with LT3 during the first 4 weeks, and patients were asked to follow a low-iodine diet during the last week before RAI administration. All high-risk patients and selective intermediate-risk group patients (online suppl. Appendix A) were prepared with THW. A second stimulation method using rhTSH (Thyrogen) was performed in all patients in the low-risk group, as well as in selective patients in the intermediate-risk group (online suppl. Appendix A), following the standard protocol: rhTSH injections on day 1 and day 2 , followed by RAI administration on day 3.

Similarly, RAI activity varied between patients depending on the postoperative risk category. No RAI ablation was performed in very low-risk patients. Subjects in the low-risk group received an activity of $30 \mathrm{mCi}$, while the intermediate-risk group received 100 mCi RAI. Finally, the high-risk group was administered a RAI activity of $150 \mathrm{mCi}$.

A diagnostic RAI WBS with SPECT-CT was performed on day 5 or day 7 after administration of 30 or $100-150$ mCi RAI, respectively, in order to inform disease staging and document the RAI avidity of any structural disease or thyroid remnant. 


\section{Laboratory Testing}

Blood chemistry with TSH, Tg, and anti-Tg Ab testing was performed on the day of RAI administration (pre-TSH, -Tg, and - Tg Ab). Only if serum TSH levels were above $30 \mathrm{mIU} / \mathrm{L}$ after THW, was stimulation considered sufficient for RAI administration. A second blood sampling with evaluation of these laboratory parameters was performed at the time of RAI WBS (post-TSH, -Tg, and $-\mathrm{Tg} \mathrm{Ab})$.

Serum TSH was tested using electrochemiluminescence immunoassay (Elecsys ${ }^{\circledR}$; Roche Diagnostics). An immunoradiometric assay kit (Thyroglobuline IRMA ${ }^{\circledR}$; CISbio) was used for serum Tg measurement with a functional sensitivity level of $0.7 \mathrm{ng} / \mathrm{mL}$. Automated anti-Tg $\mathrm{Ab}$ assays (Elecsys ${ }^{\circledR}$; Roche diagnostics) were used to detect the presence of anti-Tg antibodies.

All tests were performed in the central laboratory of the University Hospital in Leuven.

\section{Long-Term Follow-Up}

Six to 12 months after RAI administration, measurement of rhTSH-stimulated serum Tg and cervical ultrasonography were performed depending on the patient's risk for recurrent disease, response to initial treatment, and Tg status. Afterwards, patients were followed up through clinical checkup, laboratory testing, ultrasonography and, in selected cases, additional CT or MRI imaging with varying intervals from once every 3-12 months, based on the same parameters.

\section{Non-Tg-Related Variables}

Clinical variables including age at diagnosis, gender, and risk category, as well as pathologic variables (pTNM stage and tumor histology) were assessed as prognostic markers.

Tg-Related Variables

sTg values (pre-Tg) and the post-Tg/pre-Tg ratio (ratioTg) were analyzed as continuous parameters and correlated with patient outcome.

\section{Statistical Methodology}

Summary statistics are presented as means and standard deviation for continuous variables and as frequencies and percentages for categorical variables. Cox regression models were used for univariate analysis of the association between prognostic factors and outcome. Results are presented as hazard ratios (HR) with 95\% confidence intervals (CI). Analyses were performed using SAS software (version 9.4 of the SAS System for Windows).

\section{Results}

\section{Description of Cohort Characteristics}

Basic patient characteristics are represented in Table 1 . Both in group 1 and 2, the female to male ratio was approximately $2: 1$, with a mean age of $51( \pm 16.01)$ and 59.8 $( \pm 12.32)$ years, respectively. One hundred and thirtynine $(83.73 \%)$ patients had PTC, and $15(9.04 \%)$ had FTC in group 1, as opposed to $8(57.14 \%)$ and $5(35.71 \%)$ in group 2 . Two patients were diagnosed with poorly differ-
Table 1. Clinical and pathological characteristics of patients

\begin{tabular}{|c|c|c|}
\hline \multirow[t]{2}{*}{ Characteristic } & \multicolumn{2}{|l|}{ Cases (\%) } \\
\hline & $\begin{array}{l}\text { group 1 } \\
(n=166)\end{array}$ & $\begin{array}{l}\text { group } 2 \\
(n=14)\end{array}$ \\
\hline \multicolumn{3}{|l|}{ Gender } \\
\hline Male & $52(31.33)$ & $4(28.57)$ \\
\hline Female & $114(68.67)$ & $10(71.43)$ \\
\hline Age, mean $\pm S D$, years & $51 \pm 16.01$ & $59.8 \pm 12.32$ \\
\hline \multicolumn{3}{|l|}{ Histological subtype } \\
\hline PTC & $139(83.73)$ & $8(57.14)$ \\
\hline FTC & $15(9.04)$ & $5(35.71)$ \\
\hline Both & $1(0.60)$ & 0 \\
\hline Oncocytic & $10(6.02)$ & 0 \\
\hline PDTC & $1(0.60)$ & $1(7.14)$ \\
\hline \multicolumn{3}{|l|}{ Stage $(\mathrm{T})$} \\
\hline $\mathrm{T} 1$ & $63(38.41)$ & $2(14.29)$ \\
\hline $\mathrm{T} 2$ & $45(27.44)$ & $3(21.43)$ \\
\hline $\mathrm{T} 3$ & $46(28.05)$ & $5(35.71)$ \\
\hline $\mathrm{T} 4$ & $8(4.88)$ & $3(21.43)$ \\
\hline \multicolumn{3}{|l|}{ Stage $(\mathrm{N})$} \\
\hline $\mathrm{Nx}$ & $84(51.85)$ & $6(42.86)$ \\
\hline No & $40(24.69)$ & $6(42.86)$ \\
\hline N1 & $38(23.46)$ & $2(14.29)$ \\
\hline \multicolumn{3}{|l|}{ Focality } \\
\hline Unifocal & $139(83.73)$ & $13(92.86)$ \\
\hline Multifocal & $27(16.27)$ & $1(7.14)$ \\
\hline \multicolumn{3}{|l|}{ Risk category } \\
\hline Low risk & $31(18.67)$ & 0 \\
\hline Intermediate-low risk & $101(60.84)$ & $4(28.57)$ \\
\hline Intermediate-high risk & $32(19.28)$ & $3(21.43)$ \\
\hline High risk & $2(1.20)$ & $7(50.00)$ \\
\hline
\end{tabular}

PTC, papillary thyroid carcinoma; FTC, follicular thyroid carcinoma; PDTC, poorly differentiated thyroid carcinoma; $\mathrm{T}$, tumor; N, node (stages established according to TNM UICC 7th edition, 2009).

entiated thyroid cancer and 1 with synchronous PTC and FTC. Other tumor and pathological features are described in Table 1.

Based on our postoperative risk stratification, the majority of patients in group 1 were classified and treated as intermediate-low risk $(60.84 \%)$, whereas half of the patients in group 2 were considered high risk (50.00\%). Group 2 contained no low-risk subjects.

Serum pre-Tg values ranged from undetectable to $1,784.0 \mu \mathrm{g} / \mathrm{L}$ in group 1 and 1.1 to $4,779.0 \mu \mathrm{g} / \mathrm{L}$ in group 2, with mean pre-TSH levels of 120.6 and $88.4 \mathrm{mIU} / \mathrm{L}$, respectively.

With a median follow-up of 2.28 years (range, $0.16-$ 6.45 ) in group 1 and 3.36 years (range, 0.77-7.67) in 


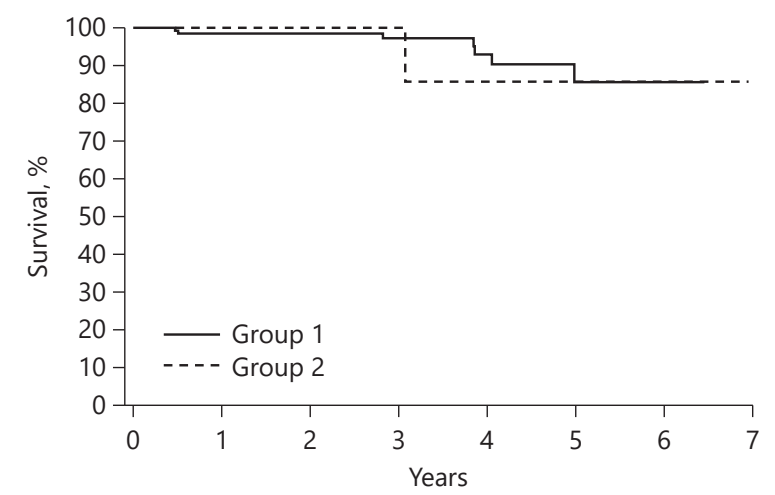

Number at risk

$\begin{array}{rrrrrrrrr}- & 166 & 131 & 94 & 65 & 36 & 18 & 7 & 0 \\ \ldots- & 14 & 12 & 10 & 7 & 4 & 3 & 2 & 1\end{array}$

Fig. 1. The Kaplan-Meier curves for overall survival in both groups.

group 2, outcome data showed a limited number of events in both analyzed groups: 5 (3.01\%) and 7 (50\%), respectively. Recurrent disease manifested locoregionally in 2 patients, as distant metastases in 1 patient and as both in 2 patients. Notably, all of these events occurred within the first 2 years following initial treatment. In group 2, disease progression was mostly seen at the site of distant metastases (5 out of 7 patients). Long-term complications of high activity RAI were seen in 6 patients, 5 of which presented with chronic sialoadenitis. One patient was diagnosed with subfertility during follow-up after RAI RRA. A total of 9 patients received at least a second RAI administration at our institution after completing initial therapy.

In our DTC cohort, there was no significant difference in overall survival rates between the two groups (Fig. 1).

\section{Predictive Markers for DFS (Group 1) and}

Progression-Free Survival (Group 2)

Table 2 shows HR for different non-Tg- and Tg-related variables with regard to risk of structural disease recurrence. Univariate analysis results confirm male gender $(\mathrm{HR}=0.09 ; p<0.05)$ and higher T-stage $(\mathrm{HR}=6.41 ; p<$ $0.05)$ as significant predictors of relapse. Both Tg-related candidate variables showed statistical significance in predicting recurrent disease: pre-Tg $(\mathrm{HR}=1.92 ; p<0.001)$ and ratioTg $(\mathrm{HR}=7.75 ; p<0.05)$. Correcting in separate bivariate models for four possible confounders (i.e., gender, age, T-stage and histology), we found that the effect of pre-Tg remained significant.
Table 2. Univariate analysis of predictive factors for structural disease recurrence (group 1) and progressive structural disease (group 2)

\begin{tabular}{llr}
\hline Variable & Hazard ratio (95\% CI) & $p$ value \\
\hline Group 1 & & \\
Gender (F vs. M) & $0.09(0.01-0.83)$ & 0.0335 \\
Age at diagnosis $(+1$ year) & $1.05(0.98-1.12)$ & 0.1552 \\
T stage (+1 level) & $6.41(1.69-24.29)$ & 0.0062 \\
Pre-Tg (×10 units $)$ & $1.92(1.41-2.62)$ & $<0.0001$ \\
ratioTg (+1 unit) & $7.75(1.85-32.51)$ & 0.0051 \\
\hline Group 2 & & \\
Gender (F vs. M) & $0.34(0.07-1.69)$ & 0.1864 \\
Age at diagnosis $(+1$ year $)$ & $0.99(0.93-1.05)$ & 0.6593 \\
T stage (+1 level) & $8.33(1.13-61.30)$ & 0.0373 \\
Pre-Tg (×10 units $)$ & $0.98(0.64-1.52)$ & 0.9395 \\
ratioTg (+1 unit) & $0.91(0.25-3.35)$ & 0.8884 \\
\hline
\end{tabular}

$\mathrm{CI}$, confidence interval; ratioTg, post-Tg/pre-Tg. Categorical variables: global $p$ value for any difference between groups is given. Binary variables: $\mathrm{HR}>(<) 1$ : increased (decreased) risk for first category. Continuous variables: $\mathrm{HR}>(<) 1$ : increased (decreased) risk with increased predictor level. ${ }^{a}$ Analysis based on log-transformed explanatory variable.

Of all candidate variables, only higher $\mathrm{T}$ stage $(\mathrm{HR}=$ $8.33 ; p<0.05)$ served to predict progressive disease.

\section{Disease Recurrence in a THW Subgroup Using \\ Different Pre-Tg Cut-Off Values}

A subgroup of patients who had received TSH stimulation in the form of THW $(n=32)$ was analyzed separately to evaluate the significance of different pre-Tg cutoff values in predicting relapse. A total of 5 subjects (15.62\%) experienced disease recurrence, which manifested locoregionally in $2(6.25 \%)$, as metastases in $1(3.13 \%)$, and as both in 2 patients (6.25\%). For pre-Tg less than 2 and $10 \mu \mathrm{g} / \mathrm{L}$, an NPV for recurrent disease of $93.33 \%$ (95\% $\mathrm{CI}=70.03-98.82)$ and $91.67 \%(95 \% \mathrm{CI}=78.74-97.03)$ was observed, respectively. Clinical outcome for this subgroup is presented in Figure 2.

\section{Discussion}

The measurement of serum $\mathrm{Tg}$ (baseline and/or stimulated) after thyroidectomy and RAI administration is a well-established tool in the monitoring of DTC patients for residual or recurrent disease during long-term follow-up [9]. Moreover, as many reports have suggested, 


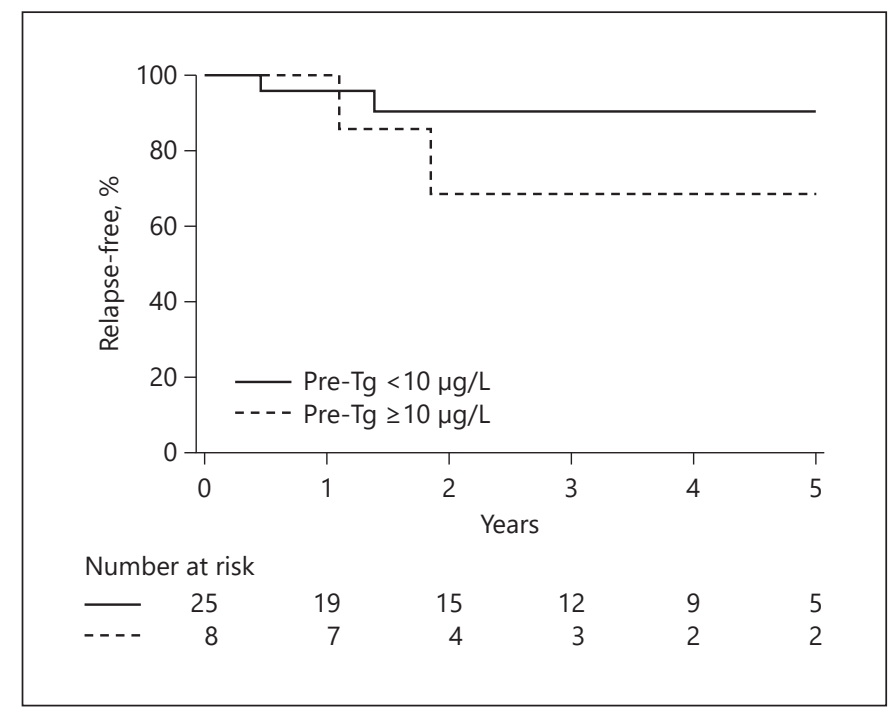

Fig. 2. Clinical outcome in the THW subgroup according to serum sTg measured at the time of RAI administration (pre-Tg). Macroscopical reappearance of disease confirmed by cytological or histopathological information was recorded as disease recurrence.

sTg values at the time of RAI administration may also be of great importance as an early prognostic marker in thyroid cancer management. This study aimed to assess pre-RAI sTg as well as immediate post-RAI Tg as a predictor of recurrent or progressive structural disease in a cohort of 180 patients treated for DTC between 2011 and 2015.

The present data show that pre-Tg is a significant predictor of relapse in patients who were considered to be structurally disease-free after thyroidectomy and RAI, the risk of recurrence almost doubling with every tenfold increase in pre-Tg. These results correspond to those reported in the literature. A meta-analysis by Webb et al. [5] including data from nearly 4,000 patients showed an overall NPV of pre-RAI sTg $<10 \mu \mathrm{g} / \mathrm{L}$ of $94.2 \%$ for predicting disease-free status. In a more recent Australian study by Matthews et al. [4], a significant difference in prognosis was found in patients with elevated $\mathrm{Tg}$ values $(>27.5 \mu \mathrm{g} / \mathrm{L})$ at the time of RAI administration. Conversely, our study could not confirm the role of serum pre-Tg as a risk factor for disease progression in patients considered to have persistent structural disease after initial therapy (group 2).

Several factors may influence $\mathrm{Tg}$ concentrations measured at the time of RAI administration, including residual thyroid tissue or tumor burden, TSH stimulation modality, functional sensitivity of Tg assays, and the presence of anti-Tg antibodies $[5,10]$. The difference in sTg between patients prepared with THW and those receiving rhTSH was demonstrated in a retrospective study by Ciappuccini et al. [10]. Apart from the notion that rhTSH may stimulate Tg production less than THW does, this effect may also be attributed to an inevitable selection bias when screening patients to receive one of both preparation modalities, that is, higher risk patients are usually prepared by means of THW. One way of dealing with this confounder is by defining separate pre- $\mathrm{Tg}$ thresholds for both TSH stimulation methods. A cut-off value for pre-RAI Tg of $>1 \mu \mathrm{g} / \mathrm{L}$ after rhTSH, suggested by Rosario et al. [11] as a negative prognostic factor, could not be tested in our cohort due to the lack of events in the low and intermediate-low risk categories. A study by Kim et al. [6] showed that dividing patients who had undergone THW into three categories based on sTg levels, that is, those with pre-RAI sTg $\leq 2 \mu \mathrm{g} / \mathrm{L}$, pre-RAI sTg between 2 and $10 \mu \mathrm{g} / \mathrm{L}$, and those with pre-RAI sTg $>10$ $\mu \mathrm{g} / \mathrm{L}$, revealed significant differences in outcome. For this reason, a subgroup analysis evaluating serum pre- $\mathrm{Tg}$ $<2$ and $<10 \mu \mathrm{g} / \mathrm{L}$ as predictors of disease recurrence was performed for only those patients in group 1 who were chosen for THW as the preferred TSH stimulation modality. The highest NPV was observed using a cut-off pre- $\mathrm{Tg}$ of $<2 \mu \mathrm{g} / \mathrm{L}$ (93.33\%). In the same subgroup, a distinction was made between locoregional and/or distant metastatic disease recurrence, since the latter has been shown to have a poorer prognosis [9]. Although Figure 1 demonstrates how pre-Tg correlates with the proportion of patients experiencing relapse, no difference was seen in location of disease recurrence with increasing pre- $\mathrm{Tg}$ values.

The lower the applied cutoff value for pre-Tg, the higher its NPV. Unfortunately, this also leads to a higher rate of false-positive results [5]. In an effort to improve the PPV of serum pre-Tg, a recent publication by Kim et al. [12] suggested taking into account the surge in Tg values noted immediately after RAI administration when assessing Tg as a predictive marker for successful ablation. Radiation-induced thyroid tissue apoptosis and subsequent destruction of membrane integrity is known to lead to a sudden release of stored Tg into the bloodstream and may therefore be a sensitive marker for early response to RAI. However, our data show that factoring in post-RAI $\mathrm{Tg}$ values (ratioTg) does not improve statistical significance of pre-RAI Tg as a risk factor for recurrent or progressive disease.

Besides its use as a prognostic tool, some authors have advocated a role for postoperative sTg in selection of pa- 
Table 3. Adverse effects of radioactive iodine treatment

\section{Acute}

Nausea

Transient sialoadenitis

Transient change in taste and smell

Subclinical abnormalities in blood count

Abnormal lacrimal function

Abnormal gonadal function (subfertility)

Chronic

Abnormal salivary function

Abnormal lacrimal function

Increased risk for second primary malignancy

tients for RAI administration, especially when combined with imaging in the form of neck ultrasonography [13] or diagnostic WBS [14]. The ultimate goal is to spare lowrisk patients from RAI, considering its possible adverse effects (Table 3). RRA has shown to be associated with a decrease in quality of life [15]. In our study, 5 patients were recorded to have developed chronic sialoadenitis. One case of subfertility was seen, though this effect could not be solely contributed to RAI.

Our research has several important limitations, the first being its small study population, in turn leading to a limited number of recorded events and therefore difficulty in achieving statistically significant results. Multivariable analysis, including all possible confounders, was not possible given the low number of events in the data. However, when the effect of pre-Tg was analyzed in separate bivariate models, correcting for gender, age, T-stage, and histology, results remained significant, suggesting that pre-Tg is an independent predictor. On the other hand, although sample size was not large, it is worth noting that patient and tumor characteristics in our series were similar to those in other reports. A second limiting factor is the length of follow-up. A substantial proportion of included patients were lost to follow-up due to them returning to the referring hospital. Nevertheless, research suggests that disease recurrence rate is at its highest during the first $2-5$ years after primary treatment, suggesting that most of the expected events in this cohort would have occurred within the presented follow-up period $[14,16,17]$. Third is the retrospective nature of this study. However, this allowed for efficient gathering of data, with results of additional diagnostic testing and their interpretation being readily available in patient files. Finally, we note that this report did not take into account biochemical disease recur- rence or persistence without structural evidence of malignancy. As research into improving prognostication in DTC patients continues, the ATA has recently introduced the concept of dynamic risk stratification. Furthermore, their guidelines suggest dividing patients into four risk strata, including a category for biochemical incomplete and indeterminate responders [9]. Yang et al. [18] demonstrated in their retrospective review of 452 cases that preablative sTg correlates with this recently developed response to therapy system, thereby allowing a prediction of long-term outcome early on during initial treatment and guiding tailored follow-up strategies. Though this was not the primary aim of the current study, confirmation of this finding in our DTC patient population may be an interesting research topic for the future.

In conclusion, our study showed that elevated pre-RAI Tg is a significant risk factor for disease recurrence in subjects with differentiated thyroid carcinoma (DTC). In a subgroup of patients prepared with THW, we observed a high NPV of a pre-Tg value of less than $10 \mu \mathrm{g} / \mathrm{L}$. Additional research is needed to investigate its role in selecting patients for RAI administration and its modalities in order to spare low-risk patients from possible adverse effects.

\section{Disclosure Statement}

The authors declare that there is no conflict of interest that could be perceived as prejudicing the impartiality of the research reported.

\section{Funding Sources}

This research did not receive any specific grant from any funding agency in the public, commercial or not-for-profit sector.
1 Sherman S: Thyroid carcinoma. Lancet 2003; 361:501-511.

2 Zubair Hussain S, Zaman M uz, Malik S, et al: Preablation stimulated thyroglobulin/TSH ratio as a predictor of successful ${ }^{131}$ remnant ablation in patients with differentiated thyroid cancer following total thyroidectomy. J Thyroid Res 2014;2014:610273.

3 Tuttle RM, Leboeuf R: Follow up approaches in thyroid cancer: a risk adapted paradigm. Endocrinol Metab Clin North Am 2008;37: 419-435, ix-x. 
4 Matthews TJ, Chua E, Gargya A, Clark J, Gao $\mathrm{K}$, Elliott M: Elevated serum thyroglobulin levels at the time of ablative radioactive iodine therapy indicate a worse prognosis in thyroid cancer: an Australian retrospective cohort study. J Laryngol Otol 2016;130(suppl 4):S50 S53.

5 Webb RC, Howard RS, Stojadinovic A, Gaitonde DY, Wallace MK, Ahmed J, Burch HB: The utility of serum thyroglobulin measurement at the time of remnant ablation for predicting disease-free status in patients with differentiated thyroid cancer: a meta-analysis involving 3947 patients. J Clin Endocrinol Metab 2012;97:2754-2763.

6 Kim TY, Kim WB, Kim ES, et al: Serum thyroglobulin levels at the time of ${ }^{131}$ Iremnant ablation just after thyroidectomy are useful for early prediction of clinical recurrence in low-risk patients with differentiated thyroid carcinoma. J Clin Endocrinol Metab 2005;90: 1440-1445.

7 Lee JI, Chung YJ, Cho BY, Chong S, Seok JW, Park SJ: Postoperative-stimulated serum thyroglobulin measured at the time of $131 \mathrm{I}$ ablation is useful for the prediction of disease status in patients with differentiated thyroid carcinoma. Surgery 2013;153:828-835.
8 Mousa U, Yilmaz AS, Nar A: Stimulated thyroglobulin values above $5.6 \mathrm{ng} / \mathrm{mL}$ before radioactive iodine ablation treatment following levothyroxine withdrawal is associated with a 2.38 -fold risk of relapse in $\mathrm{Tg}$-ab negative subjects with differentiated thyroid cancer. Clin Transl Oncol 2017;19:1028-1034.

9 Haugen BRM, Alexander EK, Bible KC, et al: 2015 American Thyroid Association Management Guidelines for Adult Patients with Thyroid Nodules and Differentiated Thyroid Cancer. Thyroid 2015;26:1-133.

10 Ciappuccini R, Hardouin J, Heutte N, Vaur D, Quak E, Rame JP, Blanchard D, de Raucourt D, Bardet S: Stimulated thyroglobulin level at ablation in differentiated thyroid cancer: the impact of treatment preparation modalities and tumor burden. Eur J Endocrinol 2014; 171:247-252.

11 Rosario PW, Siman, TL, Calsolari MR: Day 3 thyroglobulin $\leq 1 \mathrm{ng} / \mathrm{mL}$ after recombinant human TSH just prior to radioactive iodine is predictive of low risk for persistent/recurrent disease in patients with papillary thyroid carcinoma. Endocrine 2015;49:170-174.

12 Kim YI, Im HJ, Paeng JC, et al: Serum thyroglobulin level after radioiodine therapy (day 3 ) to predict successful ablation of thyroid remnant in postoperative thyroid cancer. Ann Nucl Med 2015;29:184-189.
13 Rosario PW, Mineiro Filho AF, Prates BS, Silva $\mathrm{LC}$, Calsolari MR: Postoperative stimulated thyroglobulin of less than $1 \mathrm{ng} / \mathrm{mL}$ as a criterion to spare low-risk patients with papillary thyroid cancer from radioactive iodine ablation. Thyroid 2012;22:1140-1143.

14 National Comprehensive Cancer Network 2017 NCCN Clinical Practice Guidelines in Oncology, Thyroid Carcinoma. v.1.2017:1133. https://www.nccn.org/professionals/physician_gls/pdf/thyroid.pdf.

15 Lamartina L, Durante C, Filetti S, Cooper DS: Low-risk differentiated thyroid cancer and radioiodine remnant ablation: a systematic review of the literature. J Clin Endocrinol Metab 2015;100:1748-1761.

16 Durante C, Montesano T, Torlontano M, Attard M, Monzani F, Tumino S, et al: Papillary thyroid cancer: time course of recurrences during postsurgery surveillance. J Clin Endocrinol Metab 2013;98:636-642.

17 Kim H, Kim TH, Choe JH, Kim JH, Kim JS, Oh YL, et al: Patterns of initial recurrence in completely resected papillary thyroid carcinoma. Thyroid 2017;27:908-914.

18 Yang X, Liang J, Li T, Zhao T, Lin Y: Preablative stimulated thyroglobulin correlates to new therapy response system in differentiated thyroid cancer. J Clin Endocrinol Metab 2016; 101:1307-1313. 